\title{
Complicaciones de la canalización venosa central con técnica ecoguiada y convencional en el servicio de urgencias del Hospital de San José, Bogotá, Colombia
}

Complications of the central venous catheter with ecoguide and conventional technique in emergency department of Hospital de San Jose, Bogota, Colombia

\author{
Miguel Á. Saavedra-Ortiz $M D^{1}$, \\ Ángela V. Vanegas-Pira $M D^{2}$, Oliver J. Mejía-López $M D^{3}$
}

Introducción: la utilización de la ultrasonografía como técnica de elección para la colocación de catéteres venosos centrales durante situaciones de emergencia aumenta la seguridad del paciente al presentar baja frecuencia de complicaciones, menor exposición a irradiación e incremento de la calidad de atención. Objetivo: comparar las complicaciones mecánicas de la colocación de catéter venoso central por la técnica guiada por ultrasonografía y la convencional; realizada por residentes de la especialización en Medicina de Urgencias del Hospital de San José (Bogotá, Colombia). Materiales y métodos: estudio descriptivo de dos cohortes en pacientes mayores de 15 años que ingresaron al servicio de urgencias del Hospital de San José, con indicación de colocación de catéter venoso central por técnica convencional entre 2012 y 2013 (cohorte no expuesta) y técnica ecoguiada en 2014 (cohorte expuesta). Como desenlace se observó la presencia de complicaciones en cualquiera de los dos grupos. Resultados: se incluyeron 73 casos, 38 $(52,1 \%)$ con colocación del catéter venoso central por la técnica ecoguiada. Se presentaron nueve $(12,3 \%)$ complicaciones, ocho $(88,9 \%)$ de ellas con la técnica convencional. La mediante del tiempo del procedimiento fue 20 minutos (rango

\footnotetext{
${ }^{1}$ Médico, especialista en Cirugía Vascular, especialista en Medicina de Urgencias. Instructor asociado, Fundación Universitaria de Ciencias de la Salud-Hospital de San José. Bogotá Colombia.

${ }^{2}$ Médico, especialista en Medicina de Urgencias y Paciente Crítico, Clínica Medilaser. Tunja, Colombia. Correo electrónico: vanegas810@gmail.com.

${ }_{3}^{3}$ Médico, especialista en Medicina de Urgencias y Paciente Crítico, Clínica Medilaser. Tunja, Colombia.

Conflicto de intereses: los autores declaran que no tienen conflicto de intereses

Medicina \& Laboratorio 2016; 22: 181-190

Módulo 19 (Investigación), número 44. Editora Médica Colombiana S.A. $2016^{\circ}$

Recibido el 27 de noviembre de 2016; aceptado el 01 de abril de 2016
} 
intercuatílico: 15-20 minutos) con la técnica convencional y 10 minutos (rango intercuatílico: 7-10 minutos) con la técnica ecoguiada; esta última con colocación del catéter en un único intento en el 78,9\% de los casos. Conclusión: se observó un menor número de complicaciones de tipo mecánico cuando con la técnica ecoguiada respecto a la técnica convencional en los pacientes críticos con indicación de canulación venosa central.

Palabras clave: cateterismo venoso central, ultrasonografía, complicaciones.

Introduction: The use of ultrasound as a technique of choice for central venous catheter placement during emergencies increases patient safety due to low frequency of complications, reduction of exposure to radiation, and increasing quality of care. Objective: To compare the mechanical complications of central venous catheter placement under ultrasound guided and conventional technique by residents of emergency medicine specialty at the Hospital de San Jose (Bogota, Colombia). Material and methods: Descriptive study of two cohorts, which include patients over 15 years old, admitted to the emergency department of Hospital de San Jose with indication of central venous catheter placement by conventional technique between 2012 and 2013 (unexposed cohort) and ultrasound-guided technique in 2014 (exposed cohort). As outcome was observed the presence of complications in both groups. Results: A total of 73 cases were included, 38 (52.1\%) of them with central venous catheter placement by ultrasound-guided technique. A total of nine complications $(12.3 \%)$ were presented, eight $(88.9 \%)$ of them with the conventional technique. The median of time procedure was of 20 minutes (interquartile range: 15-20 minute) with the conventional technique and 10 minutes (interquartile range: $7-10$ minutes) with ecoguide technique. In the ultrasound-guided technique, the catheter placement was achieved in an only attempt in $78.9 \%$ of cases. Conclusion: Fewer mechanical complications were observed with ultrasound guided technique respect conventional technique in critically ill patients who have indication of central venous cannulation.

Key words: Central venous catheterization, ultrasonography, complications.

Saavedra-Ortiz MA, Vanegas-Pira AV, Mejía-López OJ. Complicaciones de la canalización venosa central con técnica ecoguiada y convencional en el servicio de urgencias del Hospital de San José, Bogotá, Colombia. Medicina \& Laboratorio 2016; 22: 181-190.

$\mathrm{E}$ l abordaje de las venas centrales data de principios de siglo XX, cuando en 1927 se utilizó para cateterizar al bulbo superior de la vena yugular interna. Posteriormente, el uso clínico de la cateterización venosa central fue descrito por Aubaniac en 1952, quien resume una experiencia de 10 años con catéteres de vena subclavia para la infusión rápida de líquidos durante la reanimación de los soldados heridos en el campo de batalla [1]; desde entonces varias vías de acceso se han descrito [2]. 
La técnica descrita por Seldinger en 1953 continúa siendo utilizada para la introducción de un catéter dentro de un vaso a través de una aguja y una guía. Este procedimiento se asocia con complicaciones graves; sin embargo, es la alternativa para la canulación de las vías venosas centrales en pacientes con difícil acceso vascular periférico o requerimientos de medicación de alta osmolaridad [3].

A lo largo de la historia de la medicina se ha hecho énfasis en la seguridad del paciente, incrementando la atención de calidad, la eficiencia y la implementación de tratamientos menos invasivos y de menor irradiación, dando valor a las ventajas que ofrece la ultrasonografía y su enfoque en la medicina de urgencias [4].

Tradicionalmente el sitio de inserción de las vías venosas centrales se ubica mediante el seguimiento de diferentes referencias anatómicas; técnica que, si bien sigue estándares de colocación y es exitosa, continúa siendo una punción a ciegas y no exenta de complicaciones, entre las que se incluyen el neumotórax, el hemotórax, la punción arterial, la punción no exitosa y la embolización de guía. La frecuencia de presentación de complicaciones es de aproximadamente el 15\%, la cual depende de la experiencia del operador y de las comorbilidades del paciente $[1,2]$.

La guía ecográfica es un método soportado por la evidencia que reduce significativamente las complicaciones relacionadas con la inserción del catéter venoso central, como lo demostraron Wu y colaboradores (2013) [5] en su metanálisis y según las recomendaciones del Colegio Americano de Médicos de Emergencias (ACEP; del inglés, American College of Emergency Physicians) de 2001 [6]. No obstante, el beneficio de la ecografía no se limita a la punción venosa guiada en tiempo real.

Pitturiti y Lagreca (2015) [7] refieren que para la colocación de dispositivos de acceso venoso central se recomienda la ecografía durante la inserción del catéter venoso central para seis fines diferentes: a) evaluación ecográfica de todas las venas disponibles, b) evaluación de la vena a partir de criterios racionales basados en la ecografía, c) punción venosa guiada por ecografía en tiempo real, d) control de la guía/orientación del catéter mediante ecografía durante el procedimiento, e) control de la integridad pleuropulmonar basado en la ecografía después de la punción venosa axilar o subclavia y f) ecocardiografía transtorácica para verificar la posición de la punta del catéter al final del procedimiento.

Todo lo anterior ha generado la necesidad de conocer la experiencia local y que se demuestre la utilidad de la guía ecográfica no sólo para la ubicación del vaso, sino para el control inmediato de las complicaciones y la ubicación anatómica final del catéter. Por tal razón, el objetivo de este trabajo fue determinar la presencia de complicaciones entre la colocación de accesos venosos centrales guiados por ultrasonografía comparado con la técnica guiada por reparos anatómicos en el servicio de urgencias del Hospital de San José de la ciudad de Bogotá, Colombia. 


\section{Materiales y métodos}

\section{Diseño y población de estudio}

Se realizó un estudio descriptivo de dos cohortes, con recolección ambispectiva de la información, en el que se incluyeron pacientes mayores de 15 años que ingresaron a la sala de reanimación del servicio de urgencias de adultos del Hospital de San José (Bogotá, Colombia) durante 2012 y 2014 para la colocación de catéter venoso central de acuerdo al criterio clínico, con base en las indicaciones de: administración de medicamentos de alta osmolaridad, monitorización hemodinámica (presión venosa central-saturación venosa de oxígeno), administración de medicación vasoactiva e inotrópicos, nutrición parenteral total y como alternativa ante un acceso venoso periférico difícil [1]. Se excluyeron aquellos pacientes a quienes el catéter venoso central fue colocado por otra especialidad diferente a Urgencias.

\section{Procedimiento de colocación del catéter venoso central}

El procedimiento fue realizado por residentes de medicina de urgencias de primer, segundo y tercer año con entrenamiento previo en accesos centrales guiados por reparos anatómicos y ultrasonografía. El entrenamiento teórico-práctico en accesos venosos centrales guiados por ecografía fue dirigido por el servicio de anestesiología del hospital con una duración de 12 horas. Los residentes recibieron certificación una vez demostraron la realización de 50 procedimientos ecoguiados.

\section{Definición de cohortes y desenlace}

Se definió como cohorte no expuesta los pacientes a quienes se les colocó el catéter venoso central por reparo anatómico, teniendo en cuenta que no estaba disponible el ecógrafo durante 2012 y 2013, y como grupo expuesto los pacientes que requirieron paso de catéter ecoguiado durante 2014, utilizando el ecógrafo M-Turbo ${ }^{\circledR}$ (SonoSite, Washington, Estados Unidos).

El desenlace se definió como la presencia de complicaciones asociadas al procedimiento en cualquiera de los dos grupos, las cuales se establecieron mediante radiografía de tórax y observación directa durante el procedimiento. El seguimiento de los grupos se hizo inmediatamente posterior a la colocación del catéter venoso central hasta el éxito de la colocación o la presencia de complicaciones. Se midieron variables relacionadas con el tiempo e intentos de colocación y presencia de factores de riesgo inherentes al paciente que se relacionan con complicación en la colocación del catéter según la literatura. 


\section{Análisis estadístico y consideraciones éticas}

Las variables cualitativas se presentaron con frecuencias absolutas y relativas, y las cuantitativas con medidas de tendencia central y de dispersión, según el tipo de distribución de los datos.

Este estudio fue aprobado por el comité de ética de investigación con seres humanos del Hospital de San José.

\section{Resultados}

Se incluyeron 73 pacientes con indicación para colocación de catéter venoso central. A $35(47,9 \%)$ de ellos se les colocó el catéter venoso central por reparos anatómicos y a $38(52,1 \%)$ guiado por ecografía. Del total de pacientes $38(52,1 \%)$ eran hombres, y la edad mínima presentada fue de 20 años y la máxima de 91 años (mediana: 68 años). Con respecto a los diagnósticos de ingreso se presentó sepsis de múltiples orígenes (pulmonar, urinaria, piel, biliar) en $24(35,8 \%)$ casos y en los $49(67,1 \%)$ pacientes restantes choque cardiogénico, crisis hiperglicemias descompensadas, incluyendo cetoacidosis y estado hi-

Tabla 1. Características generales de la población $(n=73)$

$$
\text { n (\%) }
$$

Características demográficas

Hombres $38(52,1)$

Mujeres $35(47,9)$

Edad [mediana años (rango intercuartílico)]

$68(52,5-75,5)$

Diagnóstico inicial del paciente

Choque séptico $24(35,8)$

Choque cardiogénico $17(25,3)$

Cetoacidósis $7(10,4)$

Accidente cerebrovascular $\quad 3(4,4)$ hemorrágico

Trauma craneoencefálico severo $3(4,4)$

Otro $19(26,0)$

Técnica para colocación de catéter

Convencional $35(47,9)$

Ecoguiado $38(52,1)$ perosmolar, accidente cerebro vascular isquémico, hemorrágico, choque hipovolémico, hiponatremia, trauma craneoencefálico severo y pancreatitis. Las características generales de la población se presentan en la tabla 1.

La mediana del tiempo de colocación del catéter fue de 20 minutos (rango intercuartílico: 15 a 20 minutos) para la técnica convencional y de 10 minutos (rango intercuartílico; 7 a 10 minutos) para la ecoguiada. El mínimo de intentos para la colocación del catéter varió entre uno y cuatro, con una mediana de un intento (rango intercuartílico: uno a dos intentos), siendo mayor la proporción de casos con colocación del catéter en un único intento con la técnica ecoguiada respecto a la técnica convencional (78,9\% frente a $51,4 \%)$. No se obtuvo registro del número de intentos en dos pacientes con colocación por la técnica convencional y en cinco por la técnica ecoguiada (véase tabla 2). 
Las complicaciones en general se presentaron en nueve $(12,3 \%)$ casos, tres $(4,1 \%)$ debido a neumotórax, uno $(1,4 \%)$ por punción arterial, uno $(1,4 \%)$ por embolización de la guía y cuatro $(5,5 \%)$ por punción sin éxito. Del total de complicaciones, ocho $(88,9 \%)$ se presentaron bajo la técnica convencional y uno $(11,1 \%)$ con la técnica ecoguiada; este último atribuido al no éxito en la punción (véase tabla 2).

\begin{tabular}{|c|c|c|}
\hline & \multicolumn{2}{|c|}{ n (\%) } \\
\hline & Técnica convencional $(n=35)$ & Técnica ecoguiada $(n=38)$ \\
\hline \multicolumn{3}{|c|}{ Intentos para colocación del catéter } \\
\hline Uno & $18(51,4)$ & $30(78,9)$ \\
\hline Dos & $9(25,7)$ & $3(7,9)$ \\
\hline Tres & $4(11,4)$ & $0(0,0)$ \\
\hline Cuatro & $2(5,7)$ & $0(0,0)$ \\
\hline Sin registro & $2(5,7)$ & $5(13,2)$ \\
\hline \multicolumn{3}{|l|}{ Complicaciones } \\
\hline Ninguna & $27(77,1)$ & $37(97,4)$ \\
\hline Embolización & $1(2,9)$ & $0(0,0)$ \\
\hline Neumotórax & $3(8,6)$ & $0(0,0)$ \\
\hline Punción arterial & $1(2,9)$ & $0(0,0)$ \\
\hline \multirow[t]{2}{*}{ No éxito en la punción } & $3(8,6)$ & $1(2,6)$ \\
\hline & \multicolumn{2}{|c|}{ Mediana (rango intercuartílico) } \\
\hline Tiempo (minutos) & $20(15-20)$ & $10(7-10)$ \\
\hline
\end{tabular}

Del total de pacientes $29(39,7 \%)$ no tenían factores de riesgo y $44(60,3 \%)$ por lo menos un factor de riesgo. Los factores de riesgo encontrados para las complicaciones en la colocación del catéter venoso central fueron: a) coagulopatía en seis $(8,2 \%)$ casos, de los cuales uno de ellos presentó como complicación punción no exitosa, b) enfermedad pulmonar obstructiva crónica (EPOC) en cuatro $(5,5 \%)$ casos, de los cuales uno desarrolló neumotórax, uno tuvo una punción sin éxito y uno punción arterial, c) intubación orotraqueal en $22(30,1 \%)$ casos, de los cuales uno presentó punción no exitosa, d) trombocitopenia en tres $(4,1 \%)$ casos, e) alteración anatómica en dos $(2,7 \%), f)$ obesidad en tres $(4,1 \%)$; sin registro de complicaciones en estas tres últimas condiciones, y g) otro tipo de factor de riesgo en cuatro $(5,5 \%)$ casos. Los cuatro casos restantes de complicaciones no presentaron factores de riesgo.

Con referencia a los médicos en entrenamiento de medicina de urgencias, quienes realizaron los procedimientos, se registró que los residentes de primer año colocaron 28 (38,3\%) catéteres venosos centrales y los residentes de segundo y tercer año $45(61,7 \%)$. En cuanto a las complicaciones se encontraron dificultades 
en seis $(8,2 \%)$ pacientes con colocación del catéter realizada por los residentes de segundo año (tres casos de neumotórax, uno de punción arterial y dos de punciones no exitosas), dos $(2,7 \%)$ por los residentes de primer año (un caso de embolización y uno de punción sin éxito) y uno por los residentes de tercer año (un caso de punción sin éxito) (véase figura 1). Con la técnica ecoguiada sólo se presentó un caso de complicaciones en la colocación del catéter realizada por residentes de segundo año.



Figura 1. Frecuencia de complicaciones según el año de residencia de medicina de urgencia de los médicos encargados de la colocación del catéter venoso central en los pacientes estudiados $(n=73)$.

\section{Discusión}

El entrenamiento permanente en el área de la salud es el resultado del advenimiento de las nuevas tecnologías para mejorar la calidad de la atención. La utilización de nuevos dispositivos biomédicos para el diagnóstico y tratamiento de patologías en los servicios de urgencias ha permitido que los urgenciólogos tengan más herramientas para el abordaje del paciente [6].

La vía yugular interna, subclavia y femoral son venas comúnmente utilizadas; sin embargo, pueden ser técnicamente difíciles de acceder debido a las variaciones anatómicas entre los pacientes o por las cateterizaciones anteriores. Además, se ha descrito que los intentos de canulación pueden llegar a ser peligrosos e incluso mortales en ciertos casos $[5,8,9]$. De acuerdo a lo referido por Maecken y colaboradores (2007) [10] la anatomía de las estructuras vasculares varía aproximadamente en el $28 \%$ de los pacientes sometidos a canulación de vía central, por lo que la elección del sitio de acceso venoso se debe realizar con base en la experiencia personal, las necesidades clínicas y los factores de riesgo.

En el estudio de Maecken y colaboradores (2007) [10] se encontró que la canulación de la vena subclavia presenta menor frecuencia de complicaciones infecciosas y trombóticas y que, por lo tanto, esta vía puede ser la primera opción para acceder a un vaso central. Wu y colaboradores (2013) [5], en su metanálisis sugieren que 
tanto la vena yugular interna y el acceso subclavio podrían beneficiarse del uso de ultrasonografía en términos de reducción de complicaciones mecánicas y punción no exitosa.

En relación con la técnica convencional, Pittiruti y colaboradores (2000) [11], con base en los resultados de un estudio retrospectivo en más de 5.400 casos, sugieren que el abordaje lateral debajo de la vena yugular interna es la técnica más fácil y segura para la inserción percutánea de acceso venoso central, caracterizado por presentar la menor incidencia de punción arterial y neumotórax, así como una muy baja tasa de múltiples punciones.

La técnica ecoguiada o por ultrasonografía se ha venido implementando como herramienta de seguridad para identificar el vaso a canular y el trayecto de la punta de la aguja en tiempo real y a la cabecera del paciente. Feller-Kopman (2001) [12] informó que en los Estados Unidos se colocan cada año más de cinco millones de catéteres venosos centrales, con una tasa global de complicación del 15\%, entre las que se encuentran dificultades mecánicas como punción arterial y neumotórax en el $21 \%$ y hasta un $35 \%$ de intentos de inserción sin éxito.

El riesgo de complicaciones depende de varios factores, incluyendo la experiencia del operador, la urgencia de la colocación, así como aquellos propios del paciente tales como la obesidad, la alteración anatómica y la presencia de coagulopatías. En el presente estudio se pudo observar que la canulación venosa central con guía ecográfica presentó menos complicaciones mecánicas (neumotórax, hemotórax y punción arterial), mejoró el éxito de la punción en cuanto al número de intentos y el tiempo de colocación, lo que sugiere que la colocación ecoguiada presenta menos complicaciones y con menos intentos.

Mitre y colaboradores (2010) [13], en un ensayo clínico con 60 pacientes, evaluaron la cateterización venosa central de la vena yugular interna ecoguiada realizada por personal inexperto, encontrando mayor disminución de complicaciones en comparación con la técnica por reparos anatómicos, que no mostró diferencias entre los expertos y los inexpertos. En nuestro estudio los residentes recibieron entrenamiento previo y las complicaciones se encontraron en nueve (12,3\%) casos, con mayor frecuencia del tipo neumotórax (tres casos) en los procedimientos realizados por el grupo de residentes de segundo año, y con un solo caso con complicaciones ocurridas con la técnica ecoguiada. Es importante mencionar que una posible explicación para este hallazgo es que los residentes de segundo año son quienes, dado su nivel de entrenamiento, realizan el mayor número de procedimientos orientados por el residente de tercer año, lo que aumenta la probabilidad de que se presenten mayores complicaciones.

Del total de pacientes del presente estudio $44(60,3 \%)$ presentaron por lo menos un factor de riesgo, $22(30,1 \%)$ de ellos intubación orotraqueal y coagulopatía en 
seis $(8,2 \%)$, entre otros, siendo mayor la frecuencia de complicaciones en el grupo de pacientes sin ningún factor de riesgo, contrario a lo reportado en la literatura, en el que se presenta la relación entre las complicaciones y los factores de riesgo [14].

La Sociedad Americana de Anestesiólogos (ASA; del inglés, American Society of Anesthesiologists) recomienda en la guía de práctica clínica (accesos venosos centrales) la utilización de ultrasonografía para lograr altas tasas de éxito en la primera punción. En nuestro estudio se registraron hasta cuatro intentos con la técnica convencional y hasta dos con la técnica ecoguiada, observándose una disminución del número de punciones cuando se realiza el procedimiento con la técnica ecoguiada.

Lamperti y colaboradores (2012) [15] publicaron las recomendaciones basadas en la evidencia de la guía ecográfica para que sea utilizada no sólo en la canulación venosa central, sino también en la canulación periférica y arterial. Este trabajo confirma que el ultrasonido se puede utilizar con el fin de comprobar si hay complicaciones que amenazan la vida de forma inmediata después del procedimiento, así como la posición de la punta del catéter, disminuyendo el número de complicaciones de tipo mecánico.

\section{Conclusión}

En este trabajo se observó un menor número de complicaciones de tipo mecánico con la técnica de canalización venosa central ecoguiada en comparación con la técnica convencional. Además, se identificaron mejores tasas de éxito en cuanto a número de intentos y tiempo para la colocación. Los procedimientos realizados por los residentes de segundo año de medicina de urgencias presentaron mayor cantidad de complicaciones en comparación con los de primero y tercer año, y los factores de riesgo en los pacientes no presentaron asociación con la presencia de complicaciones. Recomendamos la realización de otros estudios en el que se incluyan como variables el sitio de punción (p. ej. yugular interna, externa, subclavia, femoral) y la especificación de la técnica ultrasonografía ( $p$. ej. dentro de plano o fuera de plano) y su posible relación con las complicaciones, siendo estas dos últimas factores limitantes para nuestro estudio.

\section{Bibliografía}

1. Bhullar I, Block J. Vascular Cannulation. En: Gabrielli A, Layon A, Yu M, Civetta JM, Taylor RW, Kirby RR, eds. Civetta, Taylor, \& Kirby's critical care. Philadelphia: Lippincott Williams \& Wilkins; 2009: 410-428.

2. McGee DC, Gould MK. Preventing complications of central venous catheterization. $\mathrm{N}$ Engl J Med 2003; 348: 1123-1133.

3. Cervera MF, M., Dolz M, Herráez JV, Re- mar A. Estudio clínico comparativo de tres tipos de catéteres de implantación venosa central. Med intensiva 1998; 12: 369-375.

4. Euerle B KB. Diagnostic ultrasonography in emergency medicine. Ann Emerg Med 2004; 18: 1-8.

5. Wu SY, Ling Q, Cao LH, Wang J, Xu MX, Zeng WA. Real-time two-dimensional ultrasound guidance for central venous cannula- 
tion: a meta-analysis. Anesthesiology 2013; 118: 361-375.

6. Physicians ACoE. American College of Emergency Physicians. ACEP emergency ultrasound guidelines-2001. Ann Emerg Med 2001; 38: 470-481.

7. Pittiruti M, Lagreca A. Cómo elegir el abordaje con guía ecográfica más adecuado para la inserción de una vía central: presentación del protocolo de evaluación rápida de una vena central. En: Lumb P, Karakitsos D, eds. Ecografía en medicina intensiva. España: Elsevier; 2015: 76-79.

8. Troianos CA, Hartman GS, Glas KE, Skubas NJ, Eberhardt RT, Walker JD, et al. Guidelines for performing ultrasound guided vascular cannulation: recommendations of the American Society of Echocardiography and the Society of Cardiovascular Anesthesiologists. J Am Soc Echocardiogr 2011; 24: 1291-1318.

9. Troianos CA, Hartman GS, Glas KE, Skubas NJ, Eberhardt RT, Walker JD, et al. Special articles: guidelines for performing ultrasound guided vascular cannulation: recommendations of the American Society of Echocardiography and the Society Of Cardiovascular Anesthesiologists. Anesth Analg 2012; 114: 46-72.
10. Maecken T, Grau T. Ultrasound imaging in vascular access. Crit Care Med 2007; 35: S178-185.

11. Pittiruti M, Malerba M, Carriero C, Tazza $\mathbf{L}$, Gui $\mathbf{D}$. Which is the easiest and safest technique for central venous access? A retrospective survey of more than 5,400 cases. J Vasc Access 2000; 1: 100-107.

12. Feller-Kopman D. Ultrasound-guided internal jugular access: a proposed standardized approach and implications for training and practice. Chest 2007; 132: 302-309.

13. Mitre CI, Golea A, Acalovschi I, Mocan T, Caea AM, Ruţă C, et al. Ultrasound-guided external jugular vein cannulation for central venous access by inexperienced trainees. Eur J Anaesthesiol 2010; 27: 300-303.

14. Salleras-Duran L, Fuentes-Pumarola C [Literature review on the effectiveness, complications, and user and professional satisfaction of ultrasound-guided peripheral catheterization versus traditional technique]. Enferm Clin 2015

15. Lamperti M, Bodenham AR, Pittiruti M, Blaivas M, Augoustides JG, Elbarbary M, et al. International evidence-based recommendations on ultrasound-guided vascular access. Intensive Care Med 2012; 38: 11051117. 